Beitrag des BDR

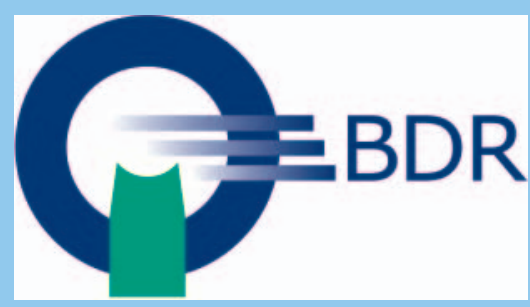

\title{
Zum Tode von Klaus Wallnöfer
}

\section{Vorkämpfer und Visionär der Radiologie}

Mit großer Trauer beklagt die Radiologie den Tod ihres langjährigen Vorsitzenden und Ehrenvorsitzenden des Berufsverbandes der Deutschen Radiologen Dr. med. Klaus Wallnöfer, München.

Jahrgang 1929, übernahm Wallnöfer nach medizinischem Staatsexamen und Weiterbildung zum Facharzt für Radiologie die radiologische Einzelpraxis seines Vaters in der Sonnenstraße im Münchner Zentrum, nahe des Stachus. Bald stießen weitere konventionelle Kollegen dazu, nächster Schritt war die Computertomografie unter Einbindung fachkundiger Kollegen. Die Zeichen der Zeit frühzeitig erkennend, gelang dann 1983 die Installation des ersten Kernspintomografen im europäischen Raum - durch das aufgeschnittene Dach der Praxis. Nun galt es, in langwierigen und schwierigen berufspolitischen Verhandlungen Kostenträger und Kassenärztliche Bundesvereinigung von der Notwendigkeit und den ganz neuen Chancen der bahnbrechenden Methode zu überzeugen. In dieser Zeit schickten viele Krankenhäuser und auch die Münchner Universitäten Patienten zur damals einzigartigen Untersuchungsmöglichkeit in Wallnöfers Praxis.

Sein Netzwerk, wie man heute sagt, war allumfassend: Delegierter der Bayerischen Landesärztekammer und der Kassenärztlichen Bundesvereinigung, stellvertretender Vorsitzender des Hartmannbundes in Bayern und der Gemeinschaft Fachärztlicher Berufsverbände (GFB). Für sein vielfältiges Engagement wurde er 1999 mit der Hartmann-Thieding-Medaille des Hartmannbundes ausgezeichnet. All diese Kontakte und Entrées galten der Hingabe an die Radiologie, verbunden mit der Überzeugung, dass nur eine entschlossene, kluge, weitsichtige und geschmeidige Berufspolitik das immer wieder infrage gestellte Fach sichern kann. Dafür ist der Berufsverband neben der primär wissenschaftlich orientierten Fachgesellschaft unverzichtbar.

Führte unser Kollege den Verband (damals noch als BVDRN, also mit Vertretung der Nuklearmedizin) wie seine Vorgänger eher von der Schreibtischecke aus, erforderten die 80er-Jahre mit all ihren standespolitischen Umbrüchen dann eine professionelle Geschäftsführung. Auch diese Entscheidung war visionär, kam doch die Radiologie mit der restriktiven Großgeräte-Bedarfsplanung bald unter massiven Druck. In flächendeckenden, vom Verband ideell und materiell unterstützten Muster-Gerichtsverfahren konnte mit der inzwischen gut laufenden Geschäftsführung und durch diese koordinierten Fachanwälten - letztlich auch verfassungsrechtlich - die Berufsausübungsfreiheit der betroffenen Radiologen durchgesetzt werden. Zwischenzeitlich band Wallnöfer - nun in eigener Sache eine weitere Praxis ohne Standortgenehmigung ein. Dies war die Geburtsstunde für die heutige Form der Großpraxis mit zahlreichen Standorten und einer Vielzahl von Mitarbeitern.

In ähnlicher Weise wurde - letztlich ebenfalls gerichtlich - die Sonografie für die Radiologie als fachgebietskonform gesichert sowie auch die Nuklearmedizin für die älteren Radiologen, bevor der Facharzt für Nuklearmedizin in der Weiterbildungsordnung geschaffen wurde. Sternstunde war die Sicherung der MRT für die zuweisungsgebundenen Fächer in der gesetzlichen Krankenversicherung, die mithilfe der Politik durchgesetzt werden konnte. Dies hat gezeigt: Mehr als andere Fächer durch staatliche und technische Regelungen wie Röntgenverordnung und ärztliche Qualitätssicherungsvorschriften geprägt und als zuweisungsgebundenes Methodenfach im Konzert der großen medizinischen Fächer, steht bei uns der Handlungs- und Beratungsbedarf auf diesen Feldern im Vordergrund. Daran hat sich nichts geändert; die von Wallnöfer installierte Verbandsführungsstruktur ist nach wie vor aktuell. Der Druck von außen auf die Radiologie in Bezug auf die MRT ist es ebenfalls.

Wallnöfer war Standespolitiker durch und durch. Seine gesellige, humorvolle Art half ihm, die Menschen und Kollegen für die Sache der Radiologie einzunehmen. Dazu gehörte auch, sich nicht allzu wichtig zu nehmen und die Einflussmöglichkeiten eines kleinen ärztlichen Verbandes nicht zu überschätzen. Aber: Fenster tun sich immer wieder auf, dann gilt es, die entscheidenden Nächte vor wichtigen Gremienversammlungen durchzustehen und die Drähte zu ziehen. Der nächste Tag ist dann meist nur noch Formsache. Wie jeder gute Politiker konnte unser Ehrenvorsitzender auch schnell seine Meinung den Erfordernissen anpassen, getreu dem alten Adenauer-Wort. Machte sich dann auf den mitunter zunächst kämpferischen Verbandssitzungen am langen Abend Müdigkeit breit, half ein - sicherer Kopfstand auf dem Sitzungstisch und es wurde wieder fröhlich und versöhnlich.

Diese vielen Aktivitäten fanden irgendwann nicht mehr das Placet der Praxispartner klassisches Problem der ärztlichen Ehrenamtlichen. Als aus dem gleichen Grund die geplante Nachfolge scheiterte, führte Wallnöfer den Verband weitere 4 Jahre bis zu seinem Ausscheiden. Zu seinem Abschied wurde jedes Vorstandsmitglied samt 
Geschäftsführern mit einem farbenfrohen Karikatur-Porträt beehrt, vom Chef mit wohlgesetzten Moritaten-Reimen zu den jeweiligen Eigenheiten am Flipchart präsentiert. Das machte ihm Spaß als von Herkunft kultureller und musischer Mensch, als Enkel eines Opernsängers in Wien und als Mitglied im akademischen Gesangsverein München. Er malte sehr gut, davon zeugen unzählige Bilder, die er bei Besu- chen gerne zeigte und bisweilen auch verschenkte. Literarisch glänzte das Multitalent im Rückblick nach seiner aktiven Zeit durch eine ausführliche, informative (und natürlich hintergründige!) Chronik des Berufsverbandes der Deutschen Radiologen, die jedes Mitglied anfordern kann.

Klaus Wallnöfer hat am 10. Juli 2021 seine stets vor Begeisterung blitzenden Augen für immer geschlossen. Wir trauern um einen weitdenkenden, klugen und engagierten Kollegen, einen humorvollen und einfach lieben Menschen, und werden ihm stets ein ehrendes Andenken bewahren.

Die Autoren:

Prof. Dr. Hermann Helmberger, München und Udo H. Cramer, Gräfelfing 